\title{
AMBIDESTRALIDADE E DESEMPENHO SOCIOAMBIENTAL DE EMPRESAS DO SETOR ELETROELETRÔNICO
}

\author{
AMBIDEXTRALITY AND THE SOCIOENVIRONMENTAL PERFORMANCE OF COMPANIES IN \\ THE ELECTRO-ELECTRONIC SECTOR \\ AMBIDIESTRALIDAD Y DESEMPEÑO SOCIOAMBIENTAL DE EMPRESAS DEL SECTOR ELECTRO ELECTRÓNICO
}

\section{RESUMO}

O objetivo deste trabalho foi estudar a relação entre a ambidestralidade e o desempenho socioambiental de organizações. Definida como a capacidade de balancear esforços entre atividades de exploitation e exploration, respectivamente fundamentadas em inovações incrementais e radicais, a ambidestralidade tem sido apontada como capacidade essencial na criação de vantagem competitiva. Estudos acerca do tema têm relacionado a ambidestralidade a desempenhos econômicos superiores, deixando lacuna no que tange às demais dimensões da sustentabilidade.
Diante da importância do tema sustentabilidade, esta pesquisa estuda o desempenho socioambiental de organizações ambidestras. Para tanto, dados obtidos em survey conduzida em 131 empresas da indústria eletroeletrônica foram submetidos à análise de cluster, análise de variância e teste post-hoc de Scheffé. Como resultado, constatou-se que as 44 organizações categorizadas como ambidestras apresentaram desempenhos ambientais e sociais superiores, evidenciando a relação positiva entre a ambidestralidade e o desempenho socioambiental.

PALAVRAS-CHAVE Inovação, sustentabilidade, organizações ambidestras, desempenho socioambiental.

Vanessa do Rocio Nahhas Scandelari vrnahhas@utfpr.edu.br

Professora do Departamento Acadêmico de Construção Civil, Universidade Tecnológica Federal do Paraná - Curitiba - PR, Brasil

João Carlos da Cunha jccunha@ufpr.br

Professor do Programa de Pós-graduação em Administração, Universidade Federal do Paraná - Curitiba - PR, Brasil

Abstract The aim of this work was to study the relationship between ambidexterity and environmental and social performance of organizations. Defined as the ability to balance efforts between exploitation and exploration activities, respectively based on incremental and radical innovations, ambidexterity has been identified as an essential capability in creating competitive advantage. Studies on the subject have been relating superior economic performance to ambidexterity, leaving a gap in relation to other dimensions of sustainability. Due to the importance that the sustainability issue has taken on in society, this research studies the environmental and social performance of ambidextrous organizations. Data obtained in a survey conducted among 131 companies in the electronics industry were subjected to Cluster Analysis, Variance Analysis and Scheffé post hoc test. As a result, it was found that the 44 ambidextrous organizations achieved the higher social and environmental performance, showing the positive relationship between ambidexterity and social and environmental performance. keywords Innovation, sustainability, ambidextrous organizations, social and environmental performance.

Resumen El objetivo de este trabajo fue estudiar la relación entre la ambidiestralidad y el desempeño socioambiental de las organizaciones. Definida como la capacidad de balancear esfuerzos entre actividades de exploitation y exploration, respectivamente fundamentadas en innovaciones incrementales y radicales, la ambidiestralidad ha sido apuntada como capacidad esencial en la creación de ventaja competitiva. Estudios acerca del tema han relacionado la ambidiestralidad a desempeños económicos superiores, dejando una laguna en lo que se refiere a las demás dimensiones de la sostenibilidad. Ante la importancia del tema sostenibilidad, esta investigación estudia el desempeño socioambiental de organizaciones ambidiestras. Para ello, los datos obtenidos en survey aplicado en 131 empresas de la industria electro electrónica fueron sometidos al análisis de cluster, análisis de variancia y test post-hoc de Scheffé. Como resultado, se constató que las 44 organizaciones categorizadas como ambidiestras presentaron desempeños ambientales y sociales superiores, evidenciando la relación positiva entre la ambidiestralidad y el desempeño socioambiental.

Palabras clave Innovación, sostenibilidad, organizaciones ambidiestras, desempeño socioambiental. 


\section{INTRODUÇÃO}

Em busca de melhores resultados econômicos, as organizações investem no desenvolvimento e no aprimoramento de tecnologias, as quais são consideradas recursos centrais e capacidades dinâmicas fundamentais à construção e à manutenção da vantagem competitiva organizacional (PENROSE, 1959; WERNERFELT, 1984; BARNEY, 1991; PETERAF, 1993; TEECE, PISANO, SHUEN, 1997). Ao despertar interesse em ambientes corporativos e acadêmicos, estudos subjacentes às relações entre tecnologia, inovação, competitividade e desempenho econômico têm-se multiplicado (PRAHALAD e HAMEL, 1990; KRUGLIANSKAS, 1996; TIGRE, 2006; ZAWISLAK e outros, 2008; SBRAGIA e outros, 2006; HAYES e outros, 2008; BOOTHBY, DUFOUR, TANG, 2010; THERRIEN, DOLOREUX, CHAMBERLIN, 2011).

Entre os estudos sobre gestão de tecnologia e inovação, encontra-se a corrente teórica que tem investigado a configuração organizacional denominada organização ambidestra, segundo a qual tanto as atividades de exploitation quanto as de exploration de tecnologia devem ser intensamente conduzidas para possibilitar a conquista de vantagem competitiva sustentável por parte das empresas (LAVIE, STETTNER, TUSHMAN, 2010). Nesse sentido, pesquisas conduzidas por Tuschman e O'Reilly (1997), O'Reilly e Tuschman (2004), He e Wong (2004), Lubatkin e outros (2006), Mom, Van den Bosch e Volberda (2009) evidenciam a relação positiva entre a ambidestralidade organizacional e os desempenhos econômicos superiores.

Sabe-se, porém, que, mediante a crescente consciência acerca do tema sustentabilidade na sociedade e dos impactos negativos decorrentes das atividades empresariais sobre esta (CMMAD, 1991; MAIA e PIRES, 2011), a competitividade das organizações não tem mais sido avaliada apenas em termos de desempenho econômico, mas também com base em aspectos sociais e ambientais, numa perspectiva de longo prazo (PORTER e LINDE, 1995; HART, 2006; NASCIMENTO, LEMOS, MELLO, 2008; BARBIERI e outros, 2010; ALIGLERI, 2011).

Nesse contexto, percebe-se que legislações tornam-se mais rígidas com relação a impactos socioambientais; o mercado acionário inclui requisitos socioambientais na avaliação de empresas, a exemplo do Índice Dow Jones de Sustentabilidade (DJSI); organizações aderem a normas voluntárias direcionadas a melhorar e padronizar níveis de segurança, condições de trabalho e prestação de contas das empresas (ALIGLERI, ALIGLERI, KRUGLIANSKAS, 2009); e modelos de gestão da produção, tais como a Produção Mais Limpa (UNEP, 1989), Ecoeficiência (WBCSD, 2000) e Produção Sustentável (VELEVA e ELLENBECKER, 2001), são cada vez mais adotados por empresas que buscam maximizar resultados e minimizar impactos socioambientais.

Frente à importância da condução de atividades de exploration e exploitation de tecnologias para a manutenção do sucesso organizacional e para o atendimento aos preceitos da sustentabilidade, este trabalho pretende ampliar a fronteira do conhecimento acerca da ambidestralidade, relacionando-a a duas das dimensões do triple botton line (ELKINGTON, 1994), a ambiental e a social. Assim, busca-se responder à seguinte questão de pesquisa: Qual a relação entre a ambidestralidade e o desempenho organizacional nas dimensões ambiental e social?

Para tanto, inicia-se o artigo com a apresentação de referencial teórico acerca dos temas ambidestralidade, gestão socioambiental e modelo de produção sustentável. Na sequência, são detalhados os procedimentos metodológicos aplicados à pesquisa, bem como são expostos e analisados os resultados obtidos junto às 131 empresas participantes da survey, encerrando-se com as conclusões e implicações para futuros estudos.

\section{ORGANIZAÇÕES AMBIDESTRAS E BALANCEAMENTO ENTRE INOVAÇÕES RADICAIS E INCREMENTAIS}

Entre os desafios vivenciados pelas empresas, encontra-se o referente à ponderação entre as ações organizacionais destinadas à sustentação do sucesso atual e aquelas que têm por objetivo a condução ao sucesso futuro (LAVIE, STETTNER, TUSHMAN, 2010). Tushman e O'Reilly (1997) comparam esse desafio à participação simultânea em dois jogos, com regras e objetivos distintos. Em um dos embates, a empresa objetiva a competitividade em curto prazo e tem como foco a eficiência operacional, resultante do alinhamento entre sua estratégia, estrutura, pessoas, cultura e processos. Paralelamente, em busca da conquista do sucesso em longo prazo, as regras do segundo jogo consistem em entender como e quando iniciar um processo de ino- 
vação revolucionário e, por consequência, uma mudança tecnológica radical na organização.

Por meio das atividades de aproveitamento de capacidades atuais, denominadas exploitation, no idioma americano, contemplam-se as ações de melhoria e eficiência em relação a produtos e processos correntes, de modo a atender às necessidades de clientes e mercados já conquistados (BENNER e TUSHMAN, 2003). As atividades de exploitation possibilitam o refinamento e a aplicação das capacidades e conhecimentos existentes, gerando inovações incrementais, viabilizando a implantação de rotinas com foco no aumento da confiabilidade de processos e no incremento dos lucros no curto prazo (MIROW, HÖLZLE, GEMÜNDEN, 2008).

As atividades de prospecção, denominadas exploration, em inglês, são destinadas à busca de novas competências e são relacionadas a retornos no longo prazo (ANDRIAPOULOS e LEWIS, 2009). Características como abertura à experimentação, flexibilidade, aceitação de riscos e interesse pelo desenvolvimento de inovações radicais apresentam-se como inerentes ao processo de prospecção e exploração de novas ideias (CHENG e VAN DE VEM, 1996).

Para mensurar as capacidades de exploration e exploitation de organizações, observa-se a presença de escalas desenvolvidas em trabalhos anteriores (BIERLY e DALY, 2001; KATILA e AHUJA, 2002; HE e WONG, 2004; Lubatkin e outros, 2006; JANSEN e outros, 2009), sendo a escala proposta por Lubaktin e outros (2006), empregada na parte empírica desta pesquisa, apresentada no Quadro 1.

As ações de exploration e de exploitation possuem objetivos distintos, porém complementares, uma vez que o sucesso de hoje não garante o sucesso de amanhã, evidenciando a necessidade da condução das duas atividades concomitantemente (BENNER e TUSHMAN, 2003). As empresas detentoras da habilidade de balancear esforços entre as ações de exploitation e de exploration foram denominadas por Duncan (1976), em seus estudos seminais acerca do tema, organizações ambidestras. Andriopoulos e Lewis (2009) salientam que esse balanceamento entre atividades relacionadas à inovação incremental e radical (de produto, processo ou organizacional) não deve ser entendido apenas como uma medíocre divisão entre duas tarefas, mas, sim, como a excelência na condução de ambas.

Percebe-se, neste ponto, a convergência entre características apresentadas pela ambidestralidade e pela sustentabilidade. Na busca pelo desenvolvimento sustentável, os modelos de gestão da produção denominados Ecoeficiência (WBCSD, 2000), Produção mais Limpa (UNEP, 1989) e Produção Sustentável (LCSP, 2001) preconizam tanto a necessidade de investimentos na melhoria de operações e tecnologias correntes (exploitation) para minimizar impactos socioambientais como a necessidade de investimentos em tecnologias radicalmente inovadoras (exploration)

\section{Quadro 1 - Escala de mensuração da intensidade de condução de atividades de exploration e exploitation}

Com que intensidade sua organização:

Busca por novas ideias tecnológicas pensando "fora da caixa", ou seja, fora dos limites da empresa.

Fundamenta o seu sucesso na sua habilidade em explorar novas tecnologias.

Atividades de Cria produtos e/ou serviços inovadores para a empresa.

exploration Busca formas criativas para satisfazer às necessidades dos clientes.

Aventura-se agressivamente em novos segmentos de mercado.

Alveja ativamente novos grupos de consumidores.

Está comprometida em melhorar a qualidade e diminuir custos.

Melhora continuamente a confiabilidade de seus produtos e serviços.

Atividades de Aumenta os níveis de automação em suas operações.

exploitation Pesquisa constantemente a satisfação dos clientes atuais.

Sintoniza suas ofertas para obter a satisfação de seus clientes correntes.

Penetra mais profundamente em sua base de clientes já existente.

Fonte: Lubatkin e outros, 2006 (tradução nossa). 
que tenham como foco o desenvolvimento sustentável.

Detecta-se, porém, que estudos acerca das organizações ambidestras têm evidenciado os desempenhos econômicos superiores alcançados por elas (HE e WONG, 2004; Lubatkin e outros, 2006; LAVIE, STETTNER e TUSHMAN, 2010; ESPALLARDO, PÉREZ e LÓPEZ, 2011), nada afirmando, no entanto, em relação ao seu desempenho ambiental e social, deixando lacuna e oportunidade para a condução desta pesquisa.

Com o objetivo de contextualizar e embasar o presente estudo acerca da relação entre a ambidestralidade e o desempenho socioambiental das organizações, além dos conceitos sobre organizações ambidestras, foram também empregados alguns dos conceitos sobre a gestão socioambiental nas organizações, os quais são expostos a seguir.

\section{GESTÃO SOCIOAMBIENTAL NAS ORGANIZAÇÕES}

Embora a preocupação com a capacidade de suporte da Terra em relação à limitação de seus recursos naturais e de seu potencial de absorção de resíduos não seja recente, percebe-se a ampliação do envolvimento governamental e corporativo com o tema sustentabilidade na segunda metade do século XX (AULICINO, 2006). Entre os trabalhos e ações seminais em prol da sustentabilidade, aos quais pode ser atribuída a mudança de postura frente aos problemas socioambientais gerados pelas atividades humanas, destacam-se os propostos por Rachel Carson (1962), Meadows (1972) e o relatório denominado Nosso Futuro Comum (CMMAD, 1991).

Frente à emergência e maior divulgação dos problemas socioambientais decorrentes do paradigma técnico-econômico dominante até o século passado, algumas legislações sobre o tema tornaram-se mais rígidas. Além das ações de comando e controle tomadas por órgãos governamentais, também agentes não governamentais, tais como o Instituto Ethos e o Conselho Empresarial Brasileiro para o Desenvolvimento Sustentável, têm conduzido fóruns de discussões que objetivam a conscientização acerca dos malefícios gerados pelo modelo de desenvolvimento até então empregado.

Em decorrência das ações supracitadas, algumas empresas passaram a incluir, em suas estratégias e práticas organizacionais, questões ambientais e sociais, além da tradicional preocupação econômica (PORTER e LINDE, 1995; ALIGLERI, 2011), atendendo ao que se conhece como Triple Botton Line (ELKINGTON, 1994).

Dessa forma, a postura mais ética e proativa assumida pelas organizações em relação à sustentabilidade, em parte, pode ser atribuída às pressões legais, e, em parte, possui fundamentos econômicos frente aos ganhos possibilitados pela aplicação eficiente de recursos e pela melhoria da imagem da empresa perante o mercado (PORTER e LINDE, 1995).

$\mathrm{Na}$ mesma linha, Nascimento, Lemos e Mello (2008) e Barbieri e outros (2010) sugerem que a vantagem competitiva das empresas, em longo prazo, somente pode ser viabilizada por meio das práticas preconizadas pelo desenvolvimento sustentável, o qual considera as questões ambientais e sociais tão importantes quanto as questões econômicas.

Também ao abordar a gestão socioambiental como questão central ao sucesso das organizações, Hart (2006) apresenta o Portfólio de Valor Sustentável, segundo o qual, em função da postura adotada pelas empresas, estas podem:

(a) aumentar seus lucros, mediante a redução de riscos e prevenção da poluição;

(b) melhorar sua reputação e imagem, por meio do manejo adequado de recursos e produtos;

(c) acelerar inovações e reposicionar-se frente ao mercado, por meio do emprego de tecnologias limpas; e

(d) cristalizar a rota do crescimento e a trajetória da empresa, por meio da visão de sustentabilidade.

Apresentando semelhanças com a visão de Hart (2006), Barbieri (2006) mostra existir, entre as empresas, uma tendência evolutiva na forma de abordar os problemas socioambientais decorrentes de sua atuação, iniciando com o controle da poluição, progredindo para a prevenção da poluição e culminando com a abordagem estratégica. As duas primeiras abordagens guardam forte relação com as exigências decorrentes de legislações, na linha da política de comando e controle. $\mathrm{Na}$ abordagem estratégica, porém, a sustentabilidade deixa de ser vista como obrigação estatutária e passa a ser reconhecida como oportunidade de negócios e fonte de vantagem competitiva para a empresa.

Para colocar em prática as estratégias e os fundamentos preconizados pelo desenvolvimento sustentável, alguns modelos de gestão da produção foram desenvolvidos. Entre os modelos que contemplam a prevenção da poluição, o manejo adequado dos recur- 
sos naturais, a adoção de tecnologias limpas, além da adequada abordagem das questões sociais e econômicas, figuram os modelos denominados Ecoeficiência, Produção Mais Limpa e Produção Sustentável, sendo esse último abordado na sequência.

\section{MODELO DE PRODUÇÃO SUSTENTÁVEL}

O modelo de Produção Sustentável foi desenvolvido pelo Lowell Center for Sustainable Production (LCSP) da Universidade de Massachusetts, buscando contemplar as dimensões ambiental, econômica e social do desenvolvimento sustentável, tendo como objetivos:

\footnotetext{
A criação de bens e serviços empregando processos e sistemas não poluentes; conservando energia e recursos naturais; viáveis economicamente; seguros e saudáveis para empregados, comunidades e consumidores; recompensando os envolvidos (VELEVA e ELLENBECKER, 2001).
}

Para operacionalizar seus objetivos, o modelo LCSP preconiza os 10 princípios descritos no Quadro 2.

O modelo de Produção Sustentável, segundo Greiner (2001), apresenta caráter evolutivo de escopo, incluindo novas variáveis e indicadores à medida que metas são alcançadas (Figura 1).
Segundo Veleva e Ellenbecker (2001, p. 524):

\begin{abstract}
Ele reflete a noção de que as organizações devem iniciar com medições simples e fáceis em relação à sua adequação às normas e em relação à sua eficiência na aplicação de recursos, movendo-se gradativamente para indicadores mais complexos que contemplem efeitos ambientais e sociais, a cadeia de fornecedores e os impactos gerados ao longo do ciclo de vida dos produtos.
\end{abstract}

O modelo de Produção Sustentável traz um conjunto de 22 indicadores acerca de práticas sustentáveis, dos quais 9 são relacionados à dimensão social e 13, à dimensão ambiental (Quadro 3).

Esse conjunto de indicadores possibilita tanto a implantação quanto o acompanhamento do desempenho empresarial em relação aos aspectos socioambientais, sendo, por esse motivo, empregado no presente trabalho, conforme será detalhado nos procedimentos metodológicos a seguir.

\section{PROCEDIMENTOS METODOLÓGICOS}

Para alcançar o objetivo de estudar a relação entre a ambidestralidade organizacional e o desempenho socioambiental das organizações, conduziu-se uma

\section{Quadro 2 - Os dez princípios do modelo de produção sustentável}

1. Produtos e embalagens são projetados para serem seguros e ecologicamente corretos durante todo o seu ciclo de vida.

2. Os serviços são organizados para satisfazer as reais necessidades humanas e promover a equidade e a justiça.

3. Resíduos e subprodutos ecologicamente incompatíveis são reduzidos, eliminados ou reciclados.

4. Substâncias químicas, agentes físicos e demais condições que apresentem riscos à saúde humana ou ao meio ambiente são eliminados.

5. Energia e materiais são conservados, e as formas de energia e materiais utilizados são os mais apropriados para os fins desejados.

6. Os locais de trabalho e as tecnologias são projetados para continuamente minimizar ou eliminar riscos físicos, químicos, biológicos e ergonômicos.

7. 0 trabalho é organizado de modo a conservar e melhorar a eficiência e a criatividade dos funcionários.

8. A segurança e o bem-estar de todos os funcionários são prioridades, bem como o contínuo desenvolvimento de seus talentos e capacidades.

9. As comunidades em torno dos locais de trabalho são respeitadas e reforçadas econômica, social, cultural e fisicamente; a equidade e a justiça são promovidas.

10. A gestão é comprometida com um amplo e aberto processo de avaliação e melhoria contínua, de modo que, em longo prazo, a viabilidade econômica da empresa ou instituição seja reforçada.

Fonte: VELEVA e ELLENBECKER, 2001. 
Figura 1 - Estrutura evolucionária de indicadores Lowell

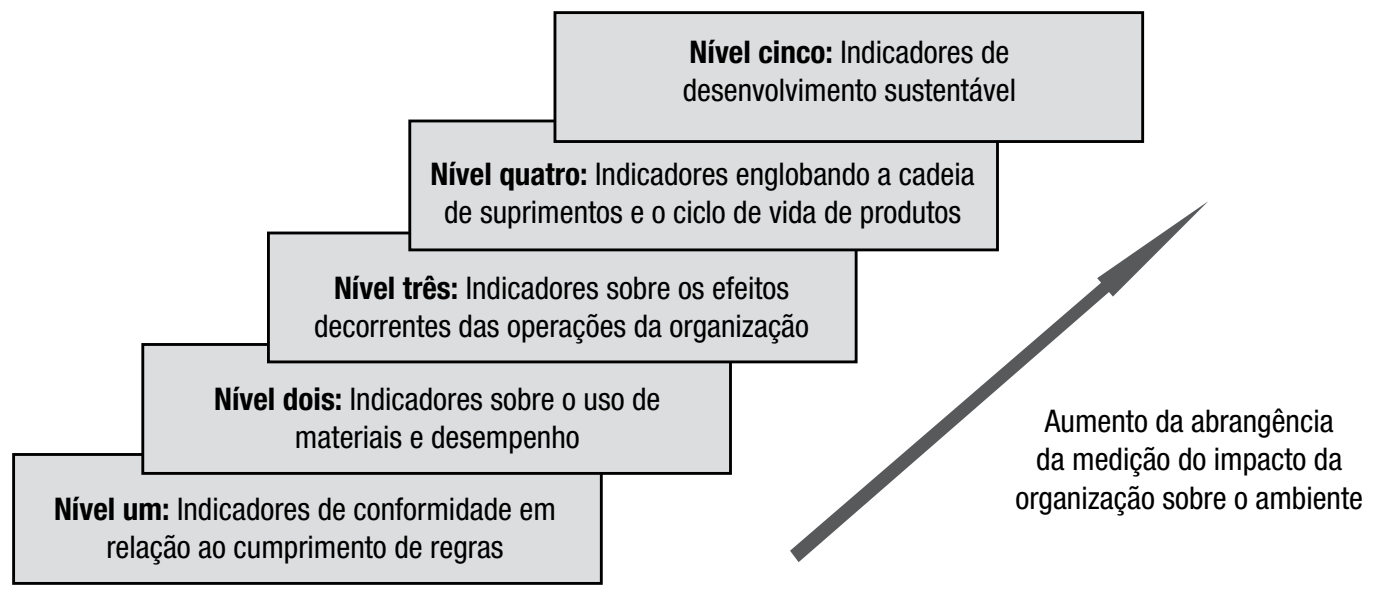

Fonte: GREINER, 2001, p. 9.

\section{Quadro 3 - Escala de mensuração da intensidade de adoção de práticas sociais e ambientais}

Dim. Com que intensidade sua organização:

Oferece treinamentos e cursos de capacitação para todos os empregados.

Busca reduzir a taxa de rotatividade da mão de obra.

Enfatiza em sua gestão as ações para evitar lesões e doenças relacionadas ao trabalho.

Implementa práticas que conduzem a melhores condições de trabalho, de modo a aumentar o bem-estar e a satisfação dos empregados com o trabalho.

SOCIAL Busca sugestões dos empregados em relação à melhoria da qualidade de produtos, processos e desempenho do sistema de saúde, segurança e meio ambiente.

Prioriza a geração de oportunidade de trabalho para a comunidade local/regional.

Adota política de inclusão de deficientes, negros, mulheres e demais grupos considerados minorias.

Realiza investimentos para a melhoria da qualidade de vida da comunidade local.

Busca envolver os stakeholders (sociedade, governo, empregados e demais partes interessadas) nas tomadas de decisões relevantes da empresa.

Busca reduzir o consumo de energia por produto.

Procura reduzir o consumo de material por produto.

Busca reduzir o consumo de água por produto.

Desenvolve ações para reduzir a quantidade de resíduos gerados em seu processo produtivo.

Difunde a prática da reciclagem em suas operações.

Oferece produtos com política take-back (retornam à empresa após o uso, para reciclagem).

AMBIENTAL Adota práticas que visam reduzir a emissão de gases (estufa e ácidos).

Desenvolve produtos com vistas a facilitar sua desmontagem, reaproveitamento e reciclagem de seus materiais (logística reversa).

Busca eliminar o emprego de materiais químicos tóxicos, persistentes e bioacumulativos.

Utiliza embalagens recicláveis/ biodegradáveis.

Busca a diminuição do uso de materiais em embalagens.

Substitui fontes de energia convencionais por energia proveniente de fontes renováveis (limpas).

Desenvolve produtos eficientes em relação ao consumo de energia, prevendo economia na fase de utilização.

Fonte: VELEVA e ELLENBECKER, 2001. 
pesquisa quantitativa (BREWER e HUNTER, 2006), descritiva (CHURCHILL, 1999) e de caráter transversal (MALHOTRA, 2001). Dessa forma, dados primários foram obtidos por meio de uma survey aplicada a uma amostra, não probabilística por adesão, de empresas do setor eletroeletrônico.

A tendência à ambidestralidade organizacional foi acessada por meio da escala desenvolvida por Lubatkin e outros (2006), a qual foi submetida a um processo de validação por meio de tradução reversa (Quadro 1). Para determinar o desempenho socioambiental das organizações, a escala empregada foi a proposta pelo modelo de Produção Sustentável (LCSP, 2001). A referida escala, além de passar pelo processo de tradução reversa, também teve sua validade de conteúdo testada com o auxílio de seis especialistas da área de sustentabilidade (Quadro 3).

Os itens das escalas supracitadas assumiram o formato do tipo Likert de 5 pontos, no qual a intensidade com que as práticas neles descritos eram conduzidas pelas organizações poderia variar entre: (1) Muito pequena, (2) Pequena, (3) Média, (4) Grande e (5) Muito grande.

Buscando-se ampliar a adesão das empresas ao levantamento, um questionário eletrônico autoaplicável foi desenvolvido sobre a plataforma Qualtrics ${ }^{\circledR}$, ficando disponível entre os dias 20 de agosto de 2010 e 25 de outubro de 2010. Entre as 533 empresas, vinculadas à Associação Brasileira da Indústria Eletroeletrônica (Abinee), as quais foram convidadas a participar da pesquisa, 168 acessaram a página eletrônica da pesquisa $(31,50 \%)$ e 134 efetivamente completaram o preenchimento das questões-chave $(25,95 \%)$. Após análise e eliminação de valores perdidos e de valores extremos (outliers), com auxílio do pacote estatístico SPSS ${ }^{\circledR}$ versão 17.0 (Statistical Package for the Social Sciences), a amostra resultou em 131 casos válidos, o que representou $24,58 \%$ da população alvo desta pesquisa.

O tratamento dos dados quantitativos teve início com a análise da adequação da amostra e da consistência interna das escalas utilizadas, ocasião em que foram efetuados os testes Alfa de Cronbach, Kaiser-Meyer-Olkin (KMO) e de esfericidade de Bartlett (HAIR e outros, 2005). Ainda nessa etapa, foram também analisadas as médias e os desvios padrão de cada um dos indicadores empregados na mensuração das capacidades de exploration e exploitation, bem como no desempenho ambiental e social das empresas participantes da pesquisa.
Na sequência, para categorizar as empresas componentes da amostra em função de suas capacidades de exploration e exploitation, foi empregada a Análise de Cluster, a qual deu origem a quatro agrupamentos, denominados: organizações ambidestras (altos escores nas práticas de exploration e exploitation), organizações com foco em exploration (altos escores exclusivamente nas práticas de exploration), organizações com foco em exploitation (altos escores exclusivamente nas práticas de exploitation) e organizações conservadoras (baixos escores em ambas as dimensões da ambidestralidade).

Finalmente, para detectar a existência de relação entre a capacidade ambidestra e o desempenho socioambiental das organizações, fez-se uso da Análise de Variância (ANOVA), seguida pelo teste post hoc de Sheffé (HAIR e outros, 2005). Por meio desses testes, pôde-se verificar se as médias de desempenho ambiental e social das quatro categorias definidas pela Análise de Cluster eram diferentes, bem como em que residia tal diferença, ou seja, qual era o agrupamento de empresas que apresentava melhores desempenhos socioambientais em relação aos demais.

\section{ANÁLISE DOS RESULTADOS}

A análise dos dados empíricos provenientes da survey foi dividida em três etapas, cujos resultados são a seguir expostos e analisados.

\section{Análise da consistência interna das escalas empregadas na pesquisa e do desempenho organizacional ambidestro, social e ambiental}

A Análise Fatorial Exploratória (AFE), realizada por meio do método dos componentes principais com rotação ortogonal Varimax (HAIR e outros, 2005), detectou, pelo critério de Kaiser (Eigenvalues), que as escalas empregadas na mensuração da ambidestralidade organizacional, desempenho social e desempenho ambiental mostraram-se bidimensionais. Dessa forma, assumiu-se: (a) a ambidestralidade como sendo composta pelas dimensões exploration e exploitation, corroborando estudos anteriores (HE e WONG, 2004; Lubatkin e outros, 2006; JANSEN e outros, 2009); (b) o desempenho social composto pelas dimensões trabalhadores (stakeholders internos) e comunidade (stakeholders externos) e (c) o desempenho ambien- 
tal como sendo composto pelas dimensões projeto (concepção) e processo (execução).

Os valores obtidos na verificação da consistência interna das escalas e na adequação da amostra obedeceram aos critérios de Hair e outros (2005), conforme expresso nas Tabelas 1, 2 e 3.

A média assumida pelos indicadores da escala adotada para apurar a ambidestralidade organizacional indica que as atividades relacionadas à dimensão exploitation, com foco em inovações incrementais e ganhos no curto prazo, são desempenhadas com maior intensidade pelas empresas investigadas, assumindo o valor $\mu=4,06$ contra $\mu=3,55$ das atividades de exploration (inovações radicais).

Na Tabela 1, percebe-se que, entre as atividades de exploration, a referente à busca por formas criativas e diferenciais para atender necessidades de clientes é a executada com maior intensidade $(\mu=3,95)$, seguida por aquelas que objetivam satisfazer novas necessidades de segmentos atuais ou novos $(\mu=3,77)$ e criar produtos/serviços inovadores para a empresa $(\mu=3,74)$. Os indicadores referentes a essas três atividades fo- ram os que apresentaram os menores valores para o desvio padrão, indicando haver certa uniformidade de comportamento entre as empresas, as quais têm o cliente como foco e se utilizam da inovação como uma das estratégias para satisfazer às necessidades destes.

$\mathrm{Na}$ extremidade oposta, o item referente à atuação agressiva em novos segmentos de mercado obteve a menor pontuação média $(2,94)$ e o maior valor para o desvio padrão, indicando que, embora, em média, as empresas se aventurem em novos segmentos de mercado com menor intensidade, existe entre elas certa heterogeneidade de comportamento em relação ao tema. Esse fato parece indicar que, mesmo num segmento reconhecidamente inovador, como o eletroeletrônico, os inovadores mais radicais são minoria, implicando valores tímidos de média para a dimensão exploration, em comparação à dimensão exploitation .

Quanto às atividades de exploitation, destaca-se que, além de obterem médias altas para seus indicadores, também apresentam menores valores de desvio padrão, indicando haver maior coesão de comportamento das empresas investigadas em relação às ativi-

\section{Tabela 1 - Dados referentes à escala de mensuração do construto ambidestralidade}

\begin{tabular}{|c|c|c|c|c|c|}
\hline Dim. & Indicador & Média & $\begin{array}{l}\text { Desvio } \\
\text { padrão }\end{array}$ & \multicolumn{2}{|c|}{ Carregamento } \\
\hline \multirow{6}{*}{ 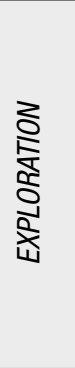 } & $\begin{array}{l}\text { Busca por novas ideéias tecnológicas pensando "fora da caixa", ou seja, fora } \\
\text { dos limites da empresa, pesquisando tecnologias diferentes das correntes. }\end{array}$ & 3,56 & 1,024 & 0,817 & 0,199 \\
\hline & $\begin{array}{l}\text { Explica o seu desempenho em função da exploração de tecnologias inovadoras, } \\
\text { ou seja, fundamenta seu sucesso na habilidade em explorar novas tecnologias. }\end{array}$ & 3,36 & 1,001 & 0,817 & 0,217 \\
\hline & Cria produtos e/ou serviços que são inovadores para a empresa. & 3,74 & 0,965 & 0,887 & 0,090 \\
\hline & $\begin{array}{l}\text { Busca formas criativas e diferenciais para satisfazer as necessidades de } \\
\text { seus clientes. }\end{array}$ & 3,95 & 0,822 & 0,681 & 0,413 \\
\hline & Aventura-se agressivamente em novos segmentos de mercado. & 2,94 & 1,101 & 0,610 & 0,036 \\
\hline & Objetiva satisfazer novas necessidades (dos segmentos atuais ou novos). & 3,77 & 0,809 & 0,568 & 0,419 \\
\hline \multirow{10}{*}{ 产 } & $\begin{array}{l}\text { Busca melhorar gradualmente a qualidade e reduzir custos de seus produtos e } \\
\text { serviços. }\end{array}$ & 4,29 & 0,728 & 0,151 & 0,692 \\
\hline & $\begin{array}{l}\text { Busca aumentar gradualmente o grau de confiabilidade de seus produtos e } \\
\text { serviços. }\end{array}$ & 4,35 & 0,655 & 0,230 & 0,740 \\
\hline & Procura ampliar os níveis de automação em suas operações. & 3,71 & 0,890 & 0,198 & 0,590 \\
\hline & Pesquisa frequentemente a satisfação dos clientes atuais. & 3,90 & 0,876 & 0,058 & 0,804 \\
\hline & $\begin{array}{l}\text { Sintoniza suas ofertas (produtos e serviços) para obter a satisfação de seus } \\
\text { clientes atuais. }\end{array}$ & 4,00 & 0,754 & 0,318 & 0,772 \\
\hline & Estreita e aprofunda relações com sua base de clientes existentes. & 4,11 & 0,816 & 0,086 & 0,737 \\
\hline & Consistência interna (Alfa de Cronbach) & & & 0,856 & 0,836 \\
\hline & Variância explicada & & & $30,1 \%$ & $29,2 \%$ \\
\hline & KM0 (Kaiser-Meyer-Olkin (KM0) & \multicolumn{4}{|c|}{0,853} \\
\hline & Teste de esfericidade de Bartlett & \multicolumn{4}{|c|}{$789,255(\mathrm{sig}=0,000)$} \\
\hline
\end{tabular}


dades de aproveitamento (Tabela 1). Entre as maiores médias obtidas nas atividades relacionadas ao construto exploitation, encontram-se os itens referentes à preocupação das empresas em relação à ampliação da qualidade e confiabilidade de seus produtos e à diminuição de seus custos, respectivamente $\mu=4,35$ e $\mu=4,29$.

No tocante ao desempenho social das organizações, os dados apresentados na Tabela 2 evidenciam que as ações relacionadas ao ambiente externo à organização, voltadas à comunidade de entorno e demais stakeholders $(\mu=3,05)$, são praticadas com menor intensidade quando comparadas àquelas voltadas ao ambiente interno $(\mu=3,84)$. Como exemplo, o indicador referente ao envolvimento de stakeholders (sociedade, governo e demais partes interessadas) nas tomadas de decisões relevantes da empresa demonstra que este é praticado com pequena ou média intensidade $(\mu=2,53)$. Esse número evidencia a pequena maturidade das empresas em relação às práticas de governança corporativa e desclosure (abertura de informações). As práticas relacionadas a investimentos com vistas à melhoria da qualidade de vida da comunidade local obtiveram média $\mu=2,69$, indicando que as organizações ainda atuam com pequena ou média intensidade nessa seara. Esse fato parece ser decorrente de aspectos culturais da sociedade brasileira, a qual atribui esse tipo de ação, primordialmente, ao governo.

Por outro lado, os indicadores relativos à responsabilidade social para com o público interno indicam que as empresas estão mais focadas em ações que, além impactarem na qualidade, produtividade e custos de produção, também atendam as diretrizes da legislação trabalhista. Exemplo disso são os altos escores atribuídos pelas empresas aos indicadores referentes à prevenção de lesões e doenças decorrentes do trabalho $(\mu=4,05)$ e à melhoria de condições e satisfação dos empregados com o trabalho $(\mu=3,88)$, práticas previstas em sistemas de gestão de saúde e segurança do trabalho e preconizadas pela norma NBR ISO 18.001.

O desempenho ambiental das organizações investigadas é retratado na Tabela 3, na qual os indicadores referentes aos processos produtivos demonstram que as empresas concentram maior esforço nas atividades de execução $(\mu=3,75)$, em comparação às de concepção $(\mu=3,23)$. Entre os 13 itens, o indicador que aborda a recuperação de produtos obsoletos para posterior tratamento e adequada disposição final, além de apre-

\section{Tabela 2 - Dados referentes à escala de mensuração do construto desempenho social}

\begin{tabular}{|c|c|c|c|c|c|}
\hline Dim. & Indicador & Média & $\begin{array}{l}\text { Desvio } \\
\text { padrão }\end{array}$ & \multicolumn{2}{|c|}{ Garregamento } \\
\hline \multirow{5}{*}{ 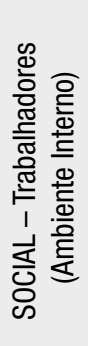 } & Oferece treinamentos e cursos de capacitação para todos os empregados. & 3,71 & 0,932 & 0,692 & 0,295 \\
\hline & Busca reduzir a taxa de rotatividade da mão de obra. & 3,82 & 0,858 & 0,778 & 0,032 \\
\hline & $\begin{array}{l}\text { Enfatiza em sua gestão as ações para evitar lesões e doenças relacionadas ao } \\
\text { trabalho. }\end{array}$ & 4,05 & 0,862 & 0,664 & 0,335 \\
\hline & $\begin{array}{l}\text { Implementa práticas que conduzem a melhores condições de trabalho, de modo } \\
\text { a aumentar o bem-estar e a satisfação dos empregados com o trabalho. }\end{array}$ & 3,88 & 0,823 & 0,833 & 0,196 \\
\hline & $\begin{array}{l}\text { Busca sugestões dos empregados em relação à melhoria da qualidade de produ- } \\
\text { tos, processos e desempenho do sistema de saúde, segurança e meio ambiente. }\end{array}$ & 3,75 & 0,939 & 0,781 & 0,138 \\
\hline \multirow{8}{*}{ 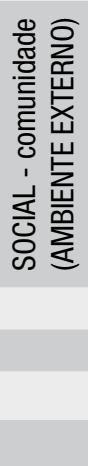 } & Prioriza a geração de oportunidade de trabalho para a comunidade local/regional. & 3,81 & 0,978 & 0,237 & 0,512 \\
\hline & $\begin{array}{l}\text { Adota política de inclusão de deficientes, negros, mulheres e demais grupos } \\
\text { considerados como minorias. }\end{array}$ & 3,16 & 1,136 & 0,051 & 0,825 \\
\hline & $\begin{array}{l}\text { Realiza investimentos para a melhoria da qualidade de vida da comunidade } \\
\text { local. }\end{array}$ & 2,69 & 1,129 & 0,228 & 0,819 \\
\hline & $\begin{array}{l}\text { Busca envolver os stakeholders (sociedade, governo, empregados e demais } \\
\text { partes interessadas) nas tomadas de decisões relevantes da empresa. }\end{array}$ & 2,53 & 1,111 & 0,155 & 0,692 \\
\hline & Consistência interna (Alfa de Cronbach) & & & 0,836 & 0,728 \\
\hline & Variância explicada & & & $33 \%$ & $26,1 \%$ \\
\hline & KM0 (Kaiser-Meyer-Olkin (KM0) & \multicolumn{4}{|c|}{0,804} \\
\hline & Teste de esfericidade de Bartlett & \multicolumn{4}{|c|}{$428,695(\mathrm{Sig}=0,000)$} \\
\hline
\end{tabular}


sentar a menor média $(\mu=2,23)$, também apresentou o maior valor de desvio padrão $(\sigma=1,395)$. Esse fato demonstra a pequena disseminação da prática take-back entre as empresas estudadas, além da heterogeneidade de comportamento frente a ela. Percebe-se alto comprometimento com a implantação de soluções por parte de empresas que atuam em mercados internacionais exigentes e regulamentados, em relação à destinação adequada dos produtos eletroeletrônicos que atingem o limite da vida útil, enquanto outras empresas da amostra nada fazem acerca do tema.

$\mathrm{Na}$ mesma linha, a questão referente à substituição de fontes de energia convencional por energia proveniente de fontes renováveis também obteve baixos valores médios $(\mu=2,56)$ e alto valor para o desvio padrão $(\sigma=1,331)$. Esse baixo valor pode ser resultante da pequena divulgação dos benefícios decorrentes do emprego de fontes de energia alternativa, da falta de regulamentação sobre o tema ou do elevado custo que estas ainda representam no curto prazo.
Os indicadores relacionados à dimensão ambiental da sustentabilidade que obtiveram maiores valores médios foram aqueles que abordam a redução de consumo de material por produto $(\mu=3,97)$ e o desenvolvimento de produtos energeticamente eficientes e econômicos $(\mu=3,95)$.

Subjacente a esses resultados, pode-se observar que as práticas ambientais mais difundidas entre as organizações são relacionadas ao atendimento à legislação, à racionalização de processos e à obtenção de legitimidade perante os clientes, em um sentido instrumental-utilitário. A título de exemplo de práticas ambientais relacionadas ao atendimento a legislações, pode-se citar a referente à eliminação do emprego de substâncias químicas tóxicas, tais como o cloro flúor carbono, os retardadores de chamas bromados (BFRs), bifenis polibrominados (PBBs) e os éteres fenil-polibrominados (PBDEs). Como exemplo de práticas ambientais com vistas à racionalização de processos e consequente ampliação de lucros,

\section{Tabela 3 - Dados referentes à escala de mensuração do construto desempenho ambiental}

\begin{tabular}{|c|c|c|c|c|c|}
\hline Dim. & Indicador & Média & $\begin{array}{l}\text { Desvio } \\
\text { padrão }\end{array}$ & \multicolumn{2}{|c|}{ Carregamento } \\
\hline \multirow{8}{*}{ 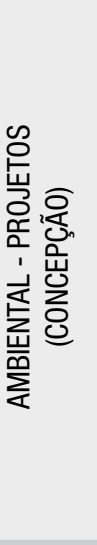 } & $\begin{array}{l}\text { Oferece produtos com política take-back (retornam à empresa após o uso, } \\
\text { para reciclagem). }\end{array}$ & 2,23 & 1,395 & 0,725 & $-0,015$ \\
\hline & Adota práticas que visam reduzir a emissão de gases (estufa e ácidos). & 3,24 & 1,307 & 0,705 & 0,312 \\
\hline & $\begin{array}{l}\text { Desenvolve produtos com vistas a facilitar sua desmontagem, reaproveita- } \\
\text { mento e reciclagem de seus materiais (logística reversa). }\end{array}$ & 3,22 & 1,204 & 0,703 & 0,300 \\
\hline & $\begin{array}{l}\text { Busca eliminar o emprego de materiais químicos tóxicos, persistentes e } \\
\text { bioacumulativos. }\end{array}$ & 3,81 & 1,164 & 0,671 & 0,298 \\
\hline & Utiliza embalagens recicláveis/ biodegradáveis. & 3,34 & 1,169 & 0,628 & 0,195 \\
\hline & Busca a diminuição do uso de materiais em embalagens. & 3,47 & 1,083 & 0,622 & 0,497 \\
\hline & $\begin{array}{l}\text { Substitui fontes de energia convencionais por energia proveniente de fontes } \\
\text { renováveis (limpas). }\end{array}$ & 2,56 & 1,331 & 0,618 & 0,298 \\
\hline & $\begin{array}{l}\text { Desenvolve produtos eficientes em relação ao consumo de energia, prevendo } \\
\text { economia na fase de utilização. }\end{array}$ & 3,95 & 0,960 & 0,571 & 0,348 \\
\hline \multirow{9}{*}{ 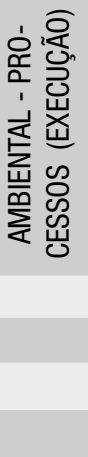 } & Busca reduzir o consumo de energia por produto. & 3,88 & 0,794 & 0,175 & 0,846 \\
\hline & Procura reduzir o consumo de material por produto. & 3,97 & 0,822 & 0,163 & 0,843 \\
\hline & Busca reduzir o consumo de água por produto. & 3,48 & 1,126 & 0,251 & 0,744 \\
\hline & $\begin{array}{l}\text { Desenvolve ações para reduzir a quantidade de resíduos gerados em seu } \\
\text { processo produtivo. }\end{array}$ & 3,67 & 0,956 & 0,386 & 0,705 \\
\hline & Difunde a prática da reciclagem em suas operações. & 3,77 & 1,113 & 0,483 & 0,500 \\
\hline & Consistência interna (Alfa de Cronbach) & & & 0,858 & 0,848 \\
\hline & Variância explicada & & & $30,5 \%$ & $26,6 \%$ \\
\hline & KM0 (Kaiser-Meyer-Olkin (KM0) & \multicolumn{4}{|c|}{0,867} \\
\hline & Teste de esfericidade de Bartlett & \multicolumn{4}{|c|}{858,55 com Sig $=0,000$} \\
\hline
\end{tabular}


pode-se citar a busca pela redução do consumo de matéria-prima, água e energia empregados tanto na composição dos produtos quanto em seu processo de produção. Finalmente, como exemplo da busca pelo reconhecimento e legitimidade perante os clientes, pode-se citar a questão da eficiência energética dos produtos eletroeletrônicos, avaliada pelos consumidores por meio do selo Procel (Programa Nacional de Conservação de Energia Elétrica), o qual impacta nas opções de compra do consumidor em função de sua classificação.

\section{Segmentação da amostra em função da tendência à ambidestralidade organizacional}

Por meio da Análise de Cluster, conduzida pelo método não hierárquico de aglomeração denominado $k$ médias ( $K$-means clustering), a amostra composta pelas 131 empresas foi segmentada em função dos escores apresentados para as capacidades de exploration e exploitation, originando os quatro grupos definidos na Figura 2.

Entre os grupos determinados pela Análise de Cluster, verificou-se que o maior deles foi o composto pelas organizações ambidestras (44 empresas $33,59 \%$ da amostra), indicando que praticamente um terço das empresas investigadas tem investido tanto em inovações incrementais, com vistas ao sucesso atual, quanto em inovações radicais, com vistas à obtenção de êxito futuro. O grupo composto por empresas cujo foco recai com maior intensidade nas atividades de exploitation, ou seja, em inovações incrementais com foco em retornos no curto prazo, foi composto por 37 empresas (28,24\%), sendo o segundo maior. As empresas com maior foco nas atividades de exploration formaram o terceiro grupo em tamanho, o qual foi composto por 31 empresas $(23,66 \%)$, indicando que um número considerável de empresas investe em atividades referentes a inovações radicais, com foco no retorno no longo prazo. O menor, entre os quatro grupos, foi o composto pelas organizações conservadoras (19 empresas $-14,50 \%$ ), evidenciando que a timidez frente a investimentos em inovações radicais e incrementais é uma posição assumida por uma pequena porção das empresas do setor eletroeletrônico participantes desta investigação.

\section{Análise da relação entre a ambidestralidade e os desempenhos social e ambiental}

Os resultados da Análise de Variância (ANOVA) expostos na Tabela 4 evidenciaram a existência de diferença estatisticamente significativa entre as médias de desempenho socioambiental apresentadas pelos grupos definidos na Análise de Cluster.

\section{Figura 2 - Resultado da análise de cluster - categorização das empresas componentes da amostra}

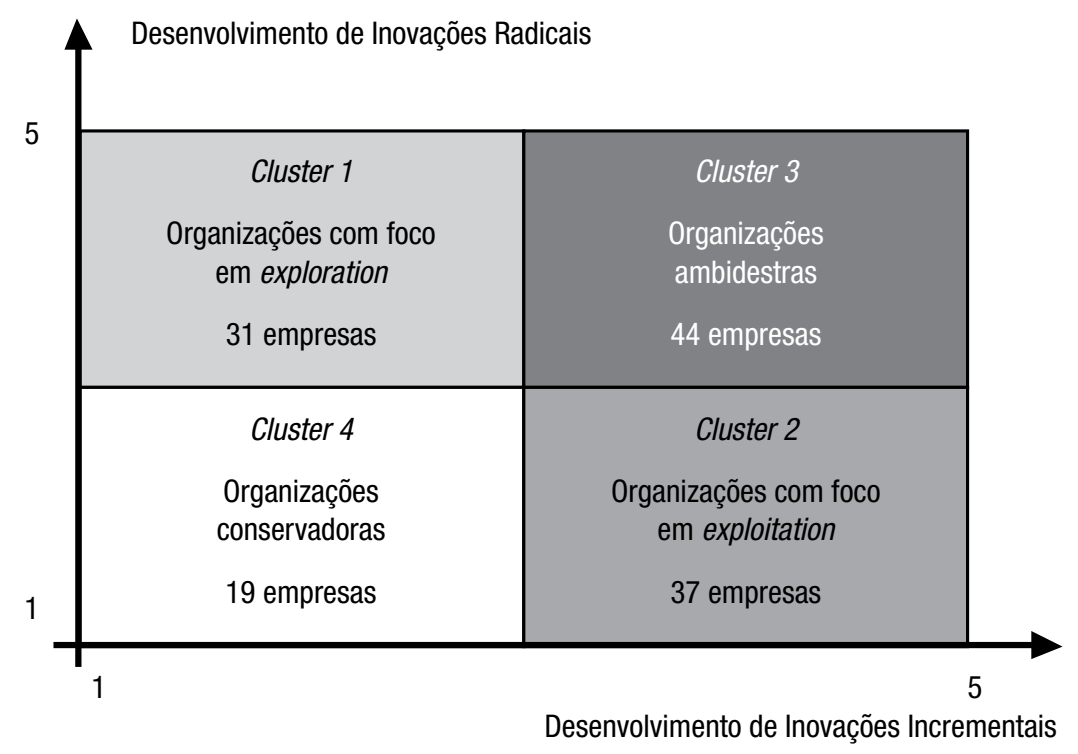


Essa constatação deu-se por meio dos valores assumidos por $\mathrm{F}$ e pelo nível de significância dos resultados, os quais apresentaram valores inferiores a 0,05 , tanto em relação ao desempenho ambiental $(\mathrm{F}(3,126)=13,33 ; \mathrm{p}<0,05)$ quanto em relação ao desempenho social $(\mathrm{F}(3,126)=14,10 ; \mathrm{p}<0,05)$. Dessa forma, para verificar entre quais grupos efetivamente ocorria a diferença de desempenho, conduziu-se o teste post-hoc de Sheffé (HAIR e outros, 2005), o qual indicou que o grupo que composto pelas empresas possuidoras dos maiores escores para as atividades de exploration e exploitation, concomitantemente, diferencia-se dos demais em relação aos desempenhos investigados. Essas evidências permitem concluir que as organizações ambidestras destacam-se por efetivamente apresentarem desempenhos organizacionais superiores, tanto na questão social quanto na ambiental, inclusive quando analisadas em relação às dimensões componentes de cada escala (Figura 3).

\section{CONCLUSÕES}

Estudos têm verificado que o adequado balanceamento entre as capacidades organizacionais de exploration e exploitation de tecnologia conduzem as empresas a desempenhos econômicos superiores (TUSCHMAN e O'REILLY, 1997; O'REILLY e TUSCHMAN, 2004; HE e WONG, 2004; MOM, VAN DEN BOSCH, VOLBERDA, 2009; LAVIE, STETTNER, TUSHMAN, 2010). Entretanto, apesar da importância que o tema sustentabilidade vem assumindo na atualidade, percebe-se uma lacuna no que tange a investigações acerca da relação entre a ambidestralidade e as demais dimensões do desenvolvimento sustentável. Nesse sentido, o presente trabalho teve como propósito estudar a relação entre a ambidestralidade e o desempenho socioambiental das organizações.

Adotando escalas anteriormente empregadas para mensurar o desempenho ambiental e social (LCSP, 2001) e as capacidades de exploration e exploration em organizações (LUBATKIN e outros, 2006), dados empíricos foram coletados por meio de uma survey conduzida em 131 empresas atuantes na indústria eletroeletrônica. Com base na pontuação atribuída pelos gerentes e diretores das empresas participantes da pesquisa ao grau com que suas organizações desenvolvem as práticas descritas nas escalas, testes estatísticos foram viabilizados.

Ao validar as escalas, como uma das contribuições desta pesquisa, corroboraram-se os estudos de He e Wong (2004); Lubatkin e outros (2006); Jansen e outros (2009) com relação à bidimensionalidade da capacidade ambidestra, formada pelas dimensões exploration e exploitation. Entre essas duas dimensões, a capacidade de exploitation, voltada ao aproveitamento das tecnologias e capacidades correntes, apresentou os maiores escores, destacando-se as atividades voltadas à ampliação do grau de confiança em produtos e serviços, seguidas pelas atividades com foco na melhoria da qualidade e na redução de custos dos mesmos. Com relação à capacidade de exploration, o maior escore foi atribuído às atividades relacionadas à busca por formas diferenciais para satisfazer as necessidades de clientes, sendo o menor escore atribuído às atividades relacionadas a aventurar-se agressivamente em novos segmentos de mercado, evidenciando que as empresas participantes desta pesquisa inovam mantendo o foco em seus clientes e mercados atuais.

As escalas empregadas na mensuração do desempenho social e ambiental das empresas também

\section{Tabela 4 - Teste de diferença entre médias de desempenho ambiental e social de empresas conservadoras, com foco em exploitation, com foco em exploration e ambidestras}

\section{ANOVA entre as médias apresentadas pelos quatro clusters nas dimensões}

Ambiental

Amb. projeto

Amb. processo

Social

Soc. trabalhadores

Soc. comunidade

OBS.: ${ }^{*} p<0,05$

\section{Sig.}

$0,000^{*}$

13,33

12,19

8,92

$0,000^{*}$

$0,000^{*}$

14,10

$0,000^{*}$

15,50

$0,000^{*}$

5,70

$0,001^{*}$ 
Figura 3 - Média dos desempenhos apresentados pelas organizações conservadoras, com foco em exploration, com foco em exploitation e ambidestras

Organizações conservadoras

Organizações com foco em exploration

Organizações com foco em exploitation

Organizações ambidestras

Organizações conservadoras

Organizações com foco em exploration

Organizações com foco em exploitation

Organizações ambidestras

Organizações conservadoras

Organizações com foco em exploration

Organizações com foco em exploitation

Organizações ambidestras
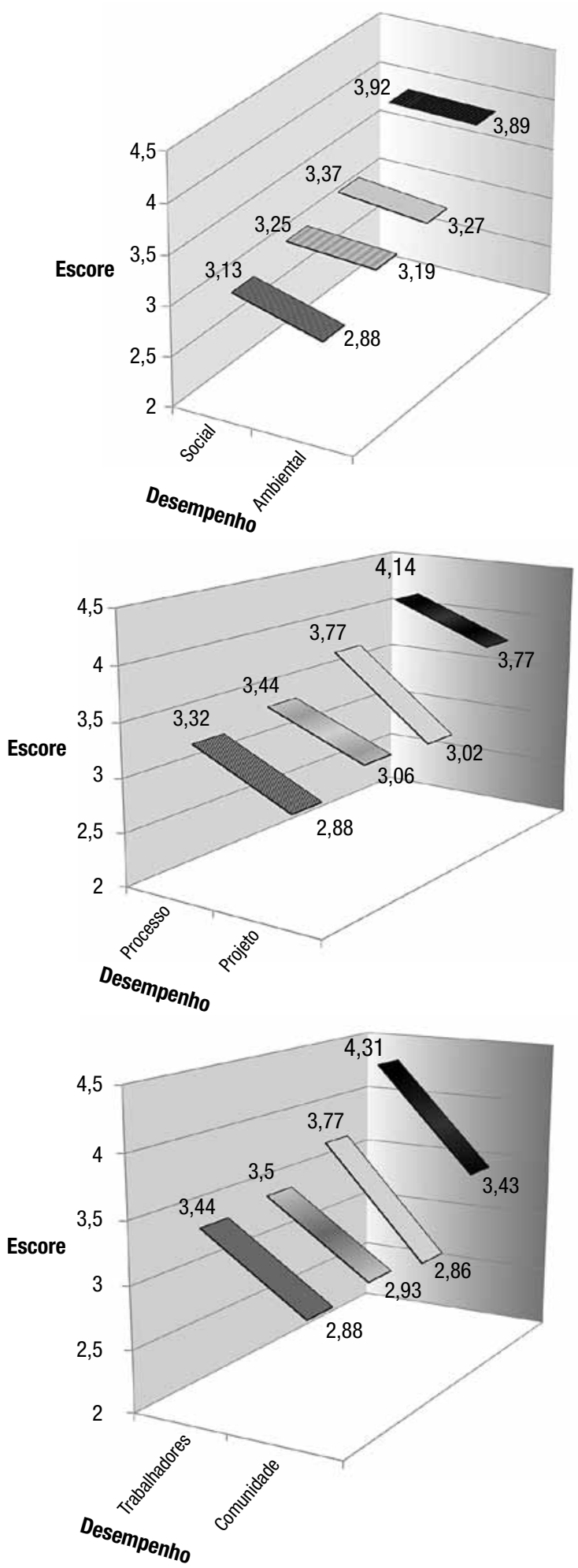
se mostraram bidimensionais, sendo o desempenho social composto pelos fatores trabalhadores (ambiente interno) e comunidade (ambiente externo), e a escala de desempenho ambiental dividida em atividades de projeto (concepção) e processo (execução). Com relação ao desempenho social, a dimensão trabalhadores, referente ao ambiente interno à organização, apresentou os maiores escores, destacando-se as atividades voltadas ao desenvolvimento de ações para evitar lesões e doenças decorrentes do trabalho e as voltadas a aumentar o bem-estar e a satisfação dos empregados com o trabalho. A dimensão comunidade obteve os menores escores, cabendo destacar o pequeno valor obtido pelo indicador referente ao envolvimento de stakeholders nas tomadas de decisões relevantes da empresa $(\mu=2,53)$, evidenciando a incipiências das ações de desclosure. Com relação ao desempenho ambiental, destacaram-se positivamente as ações voltadas à redução do consumo de material por produto produzido, ao desenvolvimento de produtos eficientes e econômicos durante o uso e à eliminação do emprego de materiais químicos tóxicos. Em outro extremo, chamou atenção a incipiência das atividades relativas à adoção de fontes energia renovável e à política take-back, na qual produtos que atingem o final de sua vida útil voltariam à empresa para tratamento e destinação adequada de resíduos $(\mu=2,23)$.

Os escores atribuídos aos indicadores de desempenho social e ambiental supracitados corroboram o raciocínio de Barbieri (2006) e Hart (2006), segundo o qual as empresas inicialmente buscam minimizar seus impactos socioambientais em função de exigências legais, passando gradativamente a ações de prevenção, até chegar à abordagem estratégica, quando a sustentabilidade deixa de ser vista como obrigação estatutária e passa a ser reconhecida como oportunidade de negócios e fonte de vantagem competitiva para a empresa.

Com relação à sua capacidade de exploration e exploitation, as 131 empresas componentes da amostra investigada foram agrupadas em função dos escores obtidos nessas dimensões, sendo que 33,6\% enquadraram-se no perfil de organizações ambidestras, inovando com grande intensidade, tanto incremental como radicalmente; $28,2 \%$ das empresas investigadas possuem maior foco nas atividades de exploitation; $23,7 \%$, nas atividades de exploration, e apenas $14,5 \%$ foram consideradas empresas conservadoras por inovarem muito pouco, fato que demonstra que as empresas do setor eletroeletrônico apresentam bom dinamismo em relação às inovações incrementais e radicais.
No que diz respeito ao desempenho ambiental e social das empresas, verificou-se que as organizações classificadas como ambidestras apresentaram os melhores resultados, sendo essas diferenças estatisticamente significativas. Dessa forma, além das constatações apresentadas em estudos anteriores acerca da superioridade do desempenho econômico obtido pelas organizações ambidestras (TUSHMAN e O'REILLY, 1997; JANSEN, VAN DEN BOSCH, VOLBERDA, 2006; MOM, VAN DEN BOSCH, VOLBERDA, 2009), pôde-se, por meio desta pesquisa, também constatar a tendência apresentada pelas organizações ambidestras com relação à obtenção de desempenhos sociais e ambientais superiores, respondendo à questão proposta por este estudo.

Embora cumprindo o objetivo de estudar a relação entre a ambidestralidade e o desempenho socioambiental em organizações do setor eletroeletrônico, este estudo traz limitações com relação à generalização estatística de seus resultados, os quais foram baseados em dados obtidos em um único setor econômico e por meio de amostra não probabilística. Dessa forma, sugere-se que a presente pesquisa seja complementada por meio de levantamentos conduzidos em outros setores industriais, preferencialmente empregando técnica de amostragem probabilística, o que possibilitaria uma generalização estatística embasada em dados mais robustos.

\section{REFERÊNCIAS}

ALIGLERI, L. M. A adoção de ferramentas de gestão para a sustentabilidade e a sua relação com os princípios ecológicos nas empresas. 2011. 170 p. Tese de Doutorado em Administração, Faculdade de Economia e Administração (FEA-USP), São Paulo, 2011.

ALIGLERI, L. M; ALIGLERI, L. A; KRUGLIANSKAS, I. Gestão socioambiental: responsabilidade e sustentabilidade do negócio. São Paulo: Atlas, 2009.

ANDRIOPOULOS, C; LEWIS, M. Exploitation-exploration tensions and organizational ambidexterity: managing paradoxes of innovation. Organization Science, v. 20, n. 4 , p. 696-717, 2009.

AULICINO, A. L. Foresight para politicas de CTEI com desenvolvimento sustentável: estudo de caso Brasil. 
2006. 322 p. Tese de Doutorado em Administração, Faculdade de Economia e Administração (FEA-USP), São Paulo, 2006.

BARBIERI, J. C. Gestão ambiental empresarial: conceitos, modelos e instrumentos. São Paulo: Saraiva, 2006.

BARBIERI, J. C; VASCONCELOS, I. F. G; ANDREASSI, T; Vasconcelos, F. C. Inovação e sustentabilidade: novos modelos e proposições. RAE-Revista de Administração de Empresas, v. 50, n. 2, p. 146-154, 2010.

BARNEY, J. Firm resources and sustained competitive advantage. Journal of Management, v. 17, n. 1, p. 99-120, 1991.

BENNER, M; TUSHMAN, M. Exploitation, exploration, and process management: the productivity dilemma revisited. Academy of Management Review, v. 28, n. 2, p. 238-274, 2003.

BIERLY, P; DALY, P. S. Exploration and exploitation in small manufacturing firms. In: 61th Annual Meeting of the Academy of Management. Anais. Washington D.C, 2001.

BOOTHBY, D; DUFOUR, A; TANG, J. Technology adoption, training and productivity performance. Research Policy, v. 39, n. 5, p. 650-661, 2010.

BREWER, J; HUNTER, A. Foundations of multimethod research: synthesizing styles. California: Sage, 2006.

CARSON, R. Silent Spring. Boston: HOUGHTON MIFFLIN. 1962.

CHENG, Y. T; VAN DE VEN, A. H. Learning the innovation journey: order out of chaos? Organization Science, v. 7 , n. 6, p. 593-614, 1996.

CHURCHILL, G. A. Marketing research: methodological foundations. 7th ed. Fort Worth: Dryden, 1999.

CMMAD - COMISSÃO MUNDIAL SOBRE MEIO AMBIENTE E DESENVOLVIMENTO. Nosso futuro comum. 2. ed. Rio de Janeiro: Fundação Getulio Vargas, 1991.

DUNCAN, R. The ambidextrous organization: designing dual structures for innovation. In: Kilman, R; Pondy, L. (Eds). The management of organizational design. New York: North Holland, 1976. p. 167-188.
ELKINGTON, J. Towards the sustainable corporation: win-win-win business strategies for sustainable development. California Management Review, v. 36, n. 2, p. 90-100, 1994.

ESPALLARDO, M. H; PÉREZ, M. S; LÓPEZ, C. S. Exploitation and exploration-based innovations: the role of knowledge in inter-firm relationships with distributors. Technovation, v. 31, n. 5, p. 203-215, 2011.

GREINER, T. Indicators of sustainable production case study: the case of Guilford of Maine Spring - 2001. Disponível em: http://www.sustainableproduction.org. Acesso em 09.10.2009.

HAIR, J; ANDERSON, R; TATHAM, R; BLACK, W. Análise multivariada de dados. 5. ed. Porto Alegre: Bookman, 2005. 593 p.

HART, S. L. O capitalismo na encruzilhada: as inúmeras oportunidades de negócios na solução dos problemas mais difíceis do mundo. Porto Alegre: Bookman, 2006.

HAYES, R; PISANO, G; UPTON, D; WHELLWRIGHT, S. Produção, estratégia e tecnologia: em busca da vantagem competitiva. Porto Alegre: Bookman, 2008.

HE, Z; WONG, P. Exploration vs. exploitation: an empirical test of the ambidexterity hypothesis. Organization Science, v. 15, n.4, p. 481-494, 2004.

JANSEN J. J. P; VAN DEN BOSCH, F. A. J; VOLBERDA, H. W. Exploratory innovation, exploitative innovation, and performance: effects of organizational antecedents and environmental moderators. Management Science, v. 52, n. 11, p. 1661-1674, 2006.

JANSEN, J. J. P; TEMPELAAR, M. P; VAN DEN BOSCH, F. A; VOLBERDA, H. W. Structural differentiation and ambidexterity: the mediating role of integration mechanisms. Organization Science, v. 20, n. 4, p. 797-811, 2009.

KATILA, R; AHUJA, G. Something old, something new: a longitudinal study of search behavior and new product introduction. Academy of Management Journal, v. 45, n. 6, p. 1183-1194, 2002.

KRUGLIANSKAS, I. Tornando a pequena e média empresa competitiva. São Paulo: Instituto de Estudos Gerenciais, 1996. 
LAVIE, D; STETTNER, U; TUSHMAN, M. Exploration and exploitation within and across organizations. The Academy of Management Annals, v. 4, n. 1, p. 109-105, 2010.

LCSP - Lowell Center for Sustainable Production - University of Massachusetts Lowell. Indicadores da produção sustentável. 2001. Disponível em: http://www.sustainableproduction. org/publ.sustainability.php?pid=146. Acesso em 15.09.2009.

LUBATKIN, M. H; SIMSEK, Z; LING, Y; VEIGA, J. F. Ambidexterity and performance in small-to medium-sized firms: the pivotal role of top management team behavioral integration. Journal of Management, v. 32, n. 5, p. 646-672, 2006.

MAIA, A. G; PIRES, P. S. Uma compreensão da sustentabilidade por meio dos níveis de complexidade das decisões organizacionais. RAM-Revista de Administração Mackenzie, v. 12, n. 3, p. 177-206, 2011.

MALHOTRA, N. K. Marketing research: an applied orientation. Upper Saddle River: Prentice-Hall, 2001.

MEADOWS, D. The limits to growth: a report for the Club of Rome's project on the predicament of man kind. New York: Universe Books, 1972.

MIROW, C. H; HÖLZLE, K; GEMÜNDEN, H. G. Putting the ambidextrous organization into practice: researching barriers to innovation within research and development. In: Annual Conference of the Academy of Management, 68, 2008, Anaheim. Anaheim: Best Paper Proceedings, 2008.

MOM, T. J. M; VAN DEN BOSCH, F. A. J; VOlBERDA, H. W. Understanding variation in managers' ambidexterity: investigating direct and interaction effects of formal structural and personal coordination mechanisms. Organization Science, v. 20, n. 4, p. 812-828, 2009.

NASCIMENTO, L. F; LEMOS, A. D. C; MELLO, M. C. A. Gestão socioambiental estratégica. Porto Alegre: Bookman, 2008.

O'REILLY, C. A.; TUSHMAN, M. L. The ambidextrous organization. Harvard Business Review, v. 82. n. 4, p. 74-81, april 2004.

PENROSE, E. The theory of the growth of the firm. 3. ed. Oxford: Oxford University, 1959.

PETERAF, M. A. The cornerstones of competitive advantage: a resource based view. Strategic Management Journal, v. 14, n. 3, p. 179-191, 1993.
PORTER, M. E; LINDE, C. Green and competitive: ending the stalemate. Harvard Business Review, v. 73, n. 5, p. 120-134, 1995.

PRAHALAD, C; HAMEL, G. The core competence of the corporation. Harvard Business Review, v. 68, n. 3, p. 7991, 1990.

SBRAGIA, R; ANDREASSI, T; CAMPANÁRIO, M. A; STAL, E. Inovação: como vencer este desafio empresarial. São Paulo: Clio, 2006.

TEECE, D. J; PISANO, G; SHUEN, A. Dynamic capabilities and strategic management. Strategic Management Journal, v. 18, n. 7, p. 509-533, 1997.

THERRIEN, P; DOLOREUX, D; CHAMBERLIN, T. Innovation novelty and (commercial) performance in the service sector: a Canadian firm-level analysis. Technovation, v. 31, n. 12, p. 655-665, 2011.

TIGRE, P. B. Gestão da inovação: a economia da tecnologia no Brasil. Rio de Janeiro: Elsevier Campus, 2006. 282 p.

TUSHMAN, M; O'Reilly, C. A. Winning through innovation: a practical guide to leading organizational change and renewal. Boston: Harvard Business School, 1997.

UNEP - United Nations Environment Programme - Division of Technology, Industry, and Economics. Sustainable consumption \& production branch: cleaner production. 1989. Disponível em: http://www.unep.fr/scp/cp. Acesso em 01.11.2009.

VELEVA, V; ELLENBECKER, M. Indicators of sustainable production: framework and methodology. Journal of Cleaner Production, v. 9, n. 6, p. 519-549, 2001.

WBCSD - World Business Council for Sustainable Development. Eco-efficiency: creating more value with less impact. 2000. Disponível em: http://www.wbcsd.org. Acesso em 11.11.2009.

WERNERFELT, B. A. A resource-based view of the firm. Strategic Management Journal, v. 5, n. 2, p. 171-180, 1984.

ZAWISLAK, P. A; BORGES, M; WEGNER,D; SANTOS, A; CASTRO-LUCAS, C. Towards the innovation function. Journal of Technology Management and Innovation, v. 3, n. 4, p. 17-30, 2008. 